\title{
Leishmania Recidivans Could Be Induced by Intralesional Infiltration of Cutaneous Leishmaniasis by Sodium Stibogluconate, with New Therapeutic Trial
}

\author{
Khalifa E Sharquie 1 (D), Raed I Jabbar ${ }^{2}$ (D)
}

${ }^{1}$ Department of Dermatology, College of Medicine, University of Baghdad, Medical City Teaching Hospital, Baghdad, Iraq

${ }^{2}$ Department of Dermatology, Fallujah Teaching Hospital, AlAnbar Health Directorate, Anbar, Iraq

\section{Correspondence: \\ Khalifa E Sharquie, Prof.}

Address: Department of

Dermatology, College of Medicine, University of Baghdad, Medical City Teaching Hospital, Baghdad, Iraq

Email: ksharquie@ymail.com

Received: 23.08.2021,

Accepted: 25.12 .2021

https://doi.org/10.29333/jcei/11516

\begin{abstract}
Objective: Leishmania recidivans is a chronic form of cutaneous leishmaniasis with characteristic picture. So the aim of this study is to record all cases of leishmania recidivans that had history of therapy of cutaneous leishmaniasis by sodium stibogluconate.

Patients and methods: This is case series descriptive study and interventional therapeutic. Twenty-two patients with clinical picture of leishmania recidivans were included. All gave a history of cutaneous leishmaniasis of 2-4-month duration before the time of therapeutic injection with sodium stibogluconate. They were treated by intralesional injections of sodium stibogluconate for several times and every two weeks and months to years before the present presentation and examination. Patients were treated by combination of oral ketoconazole, oral zinc sulfate and topical $15-25 \%$ podophyllin for around 1-2 months.
\end{abstract}

Results: Twenty-two patients were evaluated, their ages ranged from 1-20 years with a median of 10 years, with $16(72.72 \%)$ males and 6 (27.27\%) females. The location of lesions was mostly on the face and upper limbs. On examination, there were ill-defined plaques with clearing at the centers leaving some atrophy and scarring at the centers of lesions while there were active dusky red papules and nodules at periphery of primary lesion. The response to therapeutic regime was obvious after three weeks and was marked after one month and almost complete clearance at 2 months.

Conclusions: This work showed a new explanation for leishmania recidivans evolution as intralesional therapy of cutaneous leishmaniasis had induced leishmania recidivans. Oral ketoconazole and zinc sulfate combined with topical podophyllin are effective modes of treatment for leishmania recidivans.

Keywords: Cutaneous leishmaniasis, leishmania recidivans, sodium stibogluconate, ketoconazole, zinc sulfate, podophyllin

\section{INTRODUCTION}

Cutaneous leishmaniasis (CL) is a parasitic disease caused by several species of leishmania. In Iraq and other countries in Middle East, it is an endemic disease with fluctuation in their frequency, sometime reaching an epidemic state [1-3]. There are about 12 million cases worldwide, and about 1.5 million new cases of CL every year, of which over $90 \%$ present in Algeria, Afghanistan, Iraq, Iran, Syria, Saudi Arabia, Peru, and Brazil [4]. Old World disease primarily is caused by leishmania major in dry desert areas and leishmania tropica in urban areas [3]. In Iraq, two species are present: L major, the agent of zoonotic cutaneous leishmaniasis (ZCL) and L tropica, the agent of anthroponotic cutaneous leishmaniasis (ACL). Both ZCL and ACL were recorded as causative agents of cutaneous leishmaniasis in Iraq, but ACL is found predominantly in suburban areas [5].

Leishmania recidivans (LR) is a rare type of CL usually caused by leishmania tropica, and commonly follows a chronic and relapsing course. It reappears typically at the site of an original lesion that had apparently healed after a variable duration, months or years in the center or within the edge of the 
scar [6,7].

The lesion slowly progresses, often on the face, and is characterized by the appearance of recrudescing dusky red papules or nodules which form mostly around or in the site of primary healed lesions and sometime forming rings or arches. LR lesions spread slowly, persist for many years, and rarely respond to therapy, thus becoming disfiguring and destructive over the years [8].

Although leishmaniasis is a self-limiting disease that may stay for several months and even years but in some cases it might remain a chronic so called anergic leishmaniasis or in form of lupoid leishmaniasis or in form of LR where there is clearance of the center and then reappear at the same site or clearance at the center and spread peripherally. This variant of leishmaniasis has been very rare in Iraq but in the last 10 years, its frequency increased where the cause is not well recognized.

In Iraq, several studies have been carried out to diagnose leishmania parasites from cutaneous lesions of patients by using different methods including histopathological studies, direct smears examinations, cultures, serological tests $[9,10]$ and polymerase chain reaction (PCR) [11-14].

During the period from 2003-2014, four previously published studies using PCR technique for evaluation of the leishmania species in different regions of Iraq, two studies performed in two cities from the center of Iraq, Baghdad [11] and Najaf [12], L major and L tropica were the major species in both cities: in Baghdad, L major and L tropica were detected in $60 \%$ and $40 \%$, respectively of the studied patients which is comparable to what previously reported in al-Najaf city where $\mathrm{L}$ major and L tropica were detected in $56.7 \%$ and $43.3 \%$, respectively during the period 2003-2005 [12]. While in northern Iraq, Tikrit city, L tropica was the predominant species identified in $80.24 \%$ of studied sample followed by L major in $19.74 \%$ [13]. This in contrast to a study in Kut city (eastern Iraq), where L major and L tropica were detected in $70 \%$ and $18.3 \%$ of cases, respectively but no organism had been detected in $11.7 \%$ [14].

Many new topical and systemic effective therapies for CL including intralesionally injected sodium stibogluconate (SSG) were introduced [15], intralesionally injection of $7 \%$ hypertonic sodium chloride $[16,17]$, intralesionally injected $2 \%$ zinc sulfate solution $[18,19]$, topical $25 \%$ podophyllin solution [20], and oral ketoconazole and oral zinc sulfate singly and in combination [21]. Limited studies on oral ketoconazole have shown some precautions for its safety but by more recent extensive study over a huge number of patients proved its safety in treatment of different skin diseases mainly infection with dermatophytes and cutaneous leishmaniasis [22].

Thus, the aim of the present work is to gather all cases of LR aiming to find the etiopathogenesis behind this type of leishmaniasis and trying a new therapeutic combination regime.

\section{PATIENTS AND METHODS}

This is case series descriptive study and interventional therapeutic trial that had been conducted during the period from February 2017-March 2021 years. Twenty-two patients presenting with the typical features of LR were included in this study. All gave a history of CL of 2-4-month duration before the intralesional therapy with SSG. They are well documented cases of CL that had been diagnosed by history and clinical examination and confirmed by previous histopathological examinations, direct smears, cultures, serological tests, or PCR.

A thorough history was taken from each patient or his/her parents regarding the followings: age, sex, address, duration of the lesions, history of previous treatment, recurrence of the lesion, past medical history, past drug history, and obstetric history regarding the female in reproductive period.

Close physical examination was performed including site, size and sort of the lesion. Patients with the following criteria were excluded from this study: pregnancy, lactating, patients who had used any topical and systemic treatments in the previous one month, history of allergy to any medication used in our combination, unreliable patient and chronic diseases like diabetes mellitus.

The study followed the Declaration of Helsinki Principles and informed consent was taken for each patient or his/her parents before starting the therapy, after a full explaining about the nature of the disease, the method of treatment, complications, and follow up. Close-up photographs were taken before the treatment, and at each visit during the follow-up period.

All patients noticed clearance of rash at the centers of lesions but extending at the periphery. Also, all patients gave a history of treatment with repeated intralesional injections of SSG for several months to years before the present examination. While one patient had received intralesional SSG for 15 sessions followed by intramuscular SSG in a dose $20 \mathrm{mg} / \mathrm{kg} /$ day for 16 days.

Podophyllin 15-25\% was prepared by mixing 15- $25 \mathrm{~g}$ of podophyllum resin in $100 \mathrm{ml}$ tincture benzoin.

All patients with LR were treated by combination of oral ketoconazole in a dose of $200 \mathrm{mg}$ twice / day for adults while infants and children with a dose of $3.3-6.6 \mathrm{mg} / \mathrm{kg} /$ day). While oral zinc sulfate was given in a dose of $5-10 \mathrm{mg} / \mathrm{kg} /$ day in divided doses and topical 15-25\% podophyllin solution once weekly. The whole treatment regimen was given for around 1-2 months depending on response rate. The podophyllin solution was allowed to dry in about 2 minutes and patients were instructed to wash it off after 5 hours $[20,23]$.

Before treatment, one month and two months after therapy, laboratory investigations were carried out including serum aspartate aminotransferase (AST), alanine 
Table 1. Sharquie's Modified Leishmania Score to Assess the Objective Response to the Topical or Systemic Therapy

\begin{tabular}{ccccc}
\hline Score & $\begin{array}{c}\text { Change in } \\
\text { Color of } \\
\text { Lesion }\end{array}$ & $\begin{array}{c}\text { Reduction } \\
\text { Rate in Color } \\
\text { of Lesion }\end{array}$ & $\begin{array}{c}\text { Reduction } \\
\text { Rate } \\
\text { in Induration } \\
\text { of Lesion }\end{array}$ & $\begin{array}{c}\text { Reduction } \\
\text { Rate } \\
\text { of } \\
\text { Ulcer/Crust. }\end{array}$ \\
\hline $\mathbf{4}$ & Bright Red & - & - & - \\
\hline $\mathbf{3}$ & Red & $0 \%-25 \%$ & $0 \%-25 \%$ & $0 \%-25 \%$ \\
\hline $\mathbf{2}$ & Dusky Red & $25 \%-50 \%$ & $25 \%-50 \%$ & $25 \%-50 \%$ \\
\hline $\mathbf{1}$ & Dark Brown & $50 \%-75 \%$ & $50 \%-75 \%$ & $50 \%-75 \%$ \\
\hline $\mathbf{0}$ & Light Brown & $\begin{array}{c}>75 \% / \\
\text { Clearance }\end{array}$ & $\begin{array}{c}>75 \% / \\
\text { Clearance }\end{array}$ & $\begin{array}{c}>75 \% / \\
\text { Clearance }\end{array}$ \\
\hline
\end{tabular}

Score 13-16: Mild response. Score 9-12: Moderate response. Score 5-8: Marked response. Score 0-4: Complete response \& clearance. Both marked and complete responses are considered as a cure.

aminotransferase (ALT), total bilirubin (TBL), alkaline phosphatase (ALP), prothrombin time (PT), and international normalization ratio (INR). Sharquie's modified leishmania score was used to assess the response to treatment [21] and results are shown in Table 1.

Follow-up was done every week for the first month, then every two weeks for the second month, and then every month for four months to record any relapses or complications.

\section{Statistical Analysis}

Statistical package for social science (SPSS) version 22.0 was used for data input and analysis. Data were statistically described in terms of median, frequencies (no. of cases), percentage (\%), and male to female ratio.

\section{RESULTS}

Twenty-two patients were evaluated, their ages ranged from 1-20 years with a median of 10 years with $16(72.72 \%)$ males and $6(27.27 \%)$ females. The location of lesions was mostly on the face and upper limbs. The duration of lesions ranged from 0.5-4 years. All patients gave a history of treatment with intralesional injections of SSG while, one patient had received intralesional SSG for 15 sessions and there was no response, but there was central atrophy and the lesion extending peripherally. For this reason, the patient also received intramuscular injection of SSG $(20 \mathrm{mg} / \mathrm{kg} /$ day $)$ for 16 days and still, there was no response.

On examination, there was ill-defined plaques single or multiple with clearing at the center but leaving atrophy and scarring while extending centrifugally with active dusky red papules and nodules at periphery of primary lesion, sometimes forming rings or arches (Figures 1-4). The response to the therapeutic regime started after 7-10 days by decreasing in the redness and inflammatory reactions with dryness of the lesion. Obvious response was seen after three weeks and marked after one month and almost complete clearance at 2 months after starting treatment (Figures 2-4).

Regarding the percentage of the degree of response in each visit during treatment, the treatments are as follows:

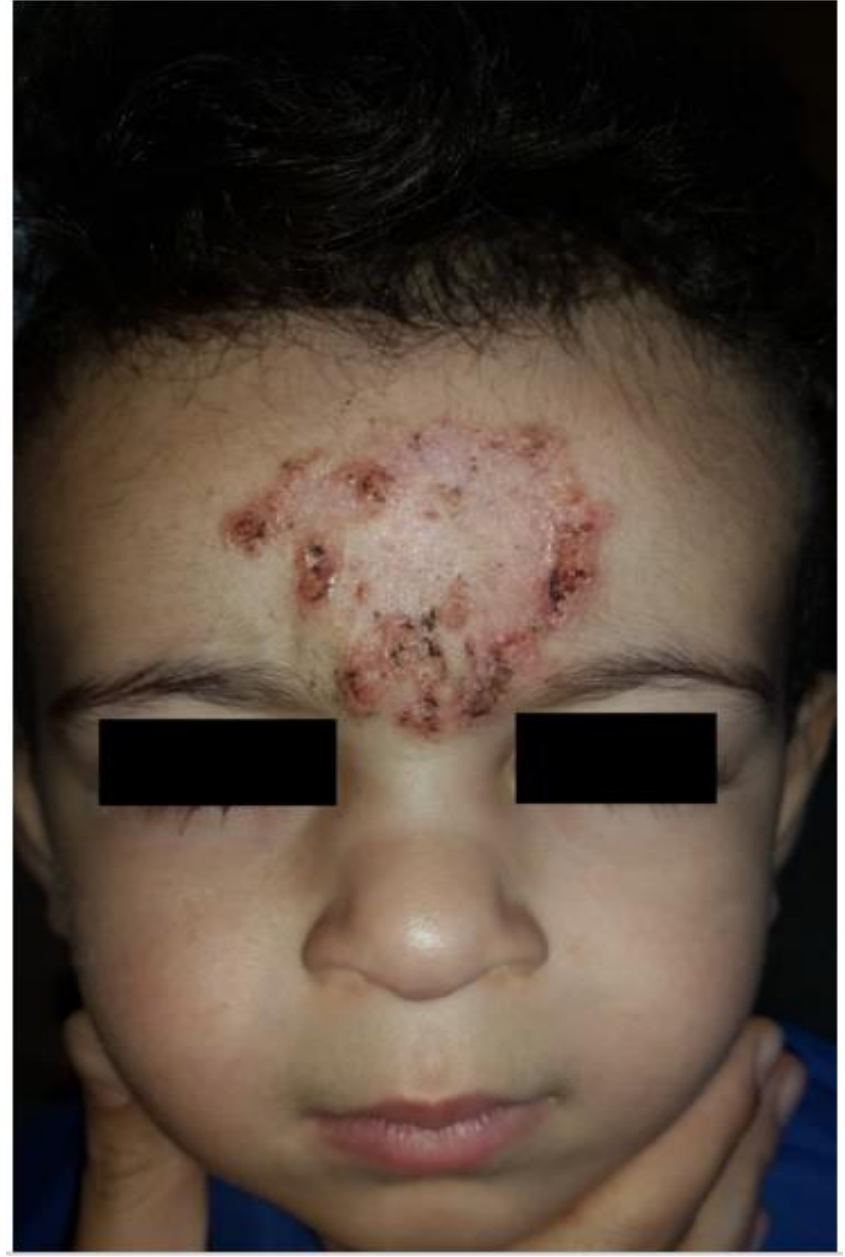

Figure 1. Three years old female with leishmania recidivans showing plaque lesion on the forehead with atrophy at the centers and active papules on the periphery

Two weeks after starting treatment: $18(81.81 \%)$ of the patients revealed mild response and $4(18.18 \%)$ revealed moderate response.

Four weeks after starting treatment: $12(54.54 \%)$ of the patients showed moderate response, 8 (36.36 \%) showed marked response, and 2(9.09\%) showed complete clearance.

Six weeks after starting treatment: $4(18.18 \%)$ patients were moderately improved, 9 (40.9\%) patients showed marked response, and 9(40.9\%) patients showed complete clearance.

Eight weeks after starting treatment: Most of the patients showed marked and complete clearance of the lesions.

The patients have well tolerated this combination and no serious systemic side effects from this combination were recorded apart from mild transient increase in serum AST and ALT was seen in $4.54 \%$ of patients, while $18.18 \%$ of treated patients with topical podophyllin solution recorded mild transient burning pain at site of lesion and these adverse effects subsided with over time. No any clinical relapse was displayed by any patient during the follow-up period. 


\section{New Therapeutic Trial for Leishmania Recidivans}

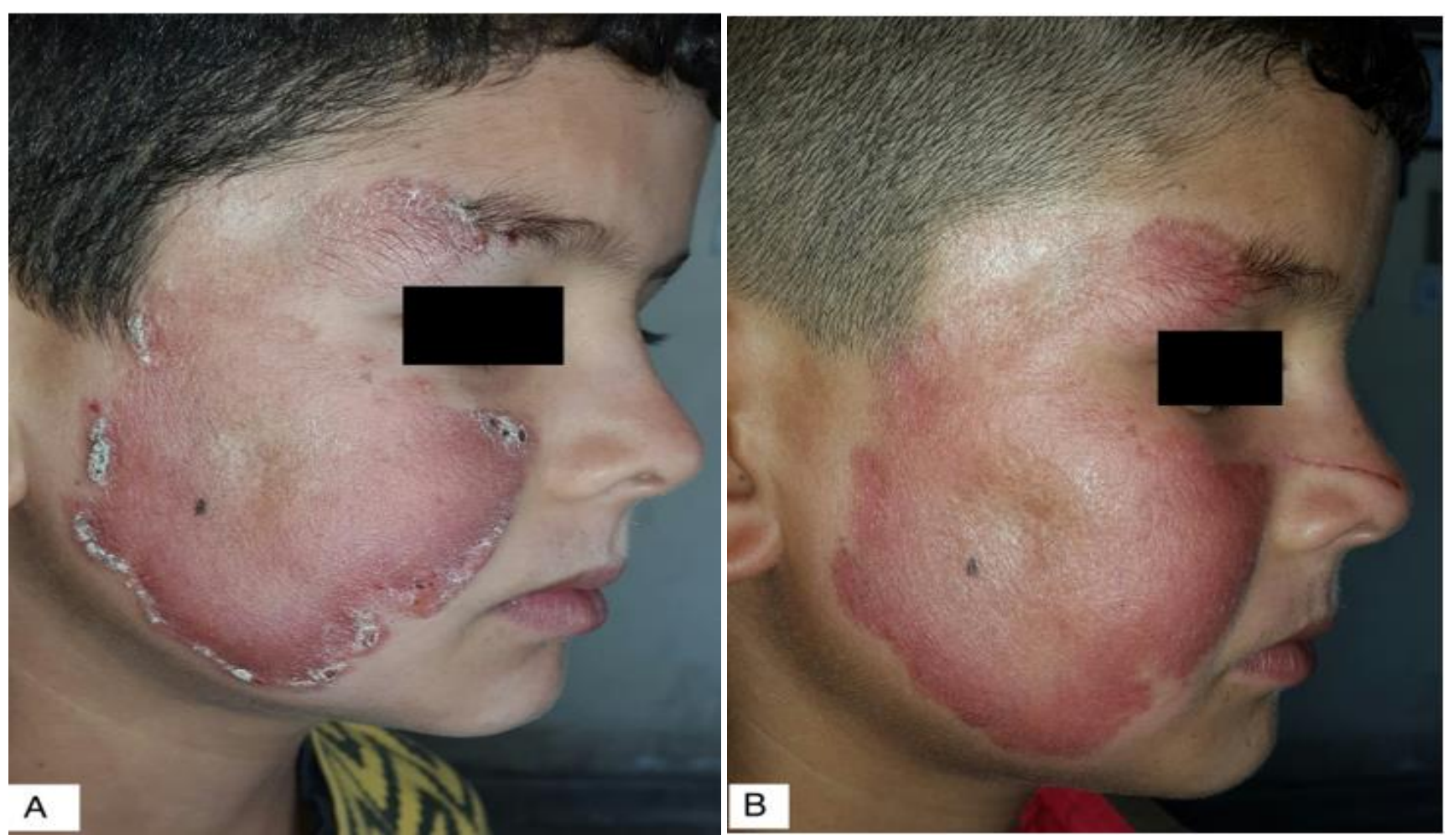

Figure 2. Eight years old male with leishmania recidivans lesion on the right side of the face showing large erythematous atrophic patch with active border: (A) before treatment and (B) the same patient two months after treatment showing complete clearance of active lesions
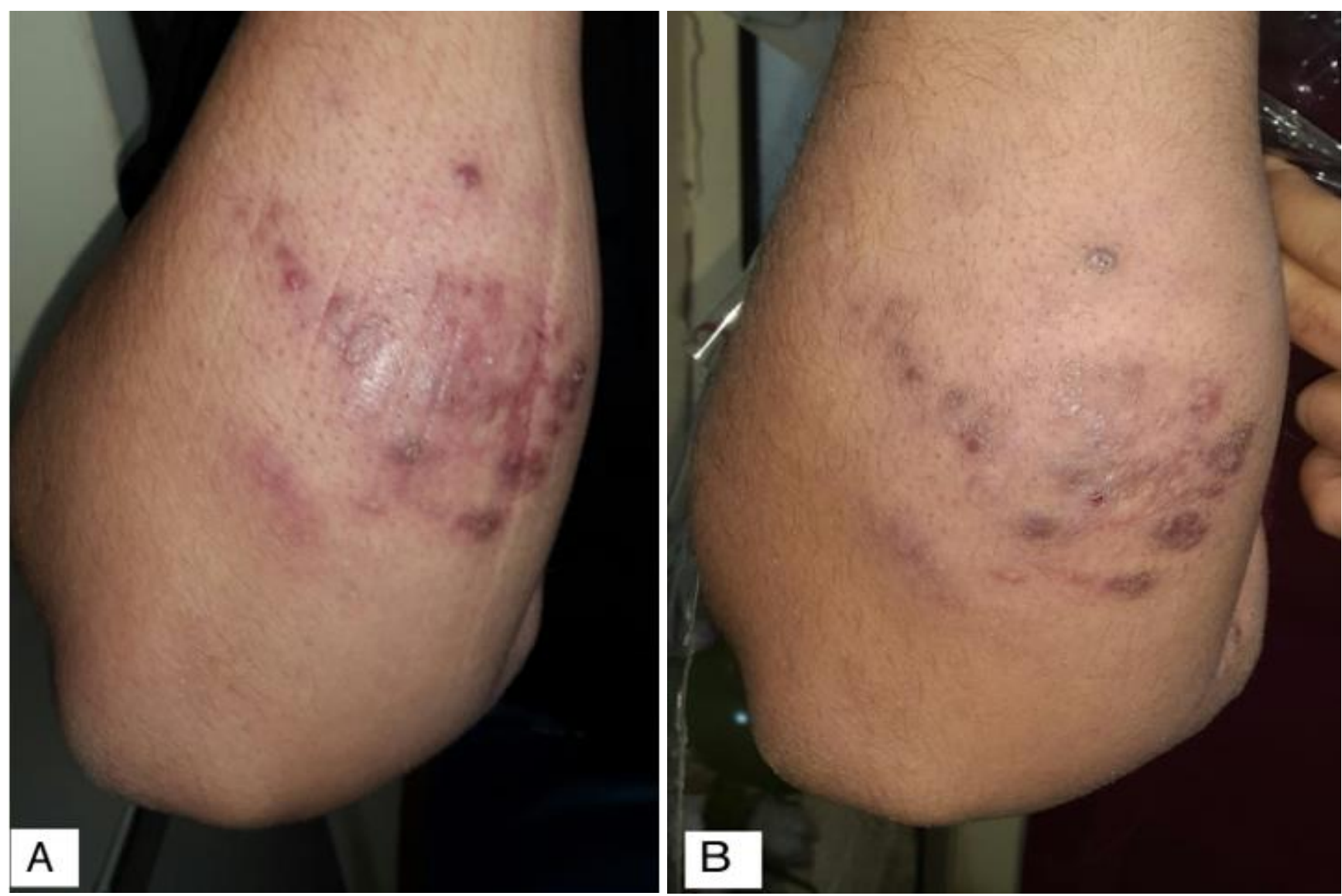

Figure 3. Twenty years old male with leishmania recidivans lesion on the right forearm: (A) before treatment and (B) two weeks after treatment showing involution of active lesions 


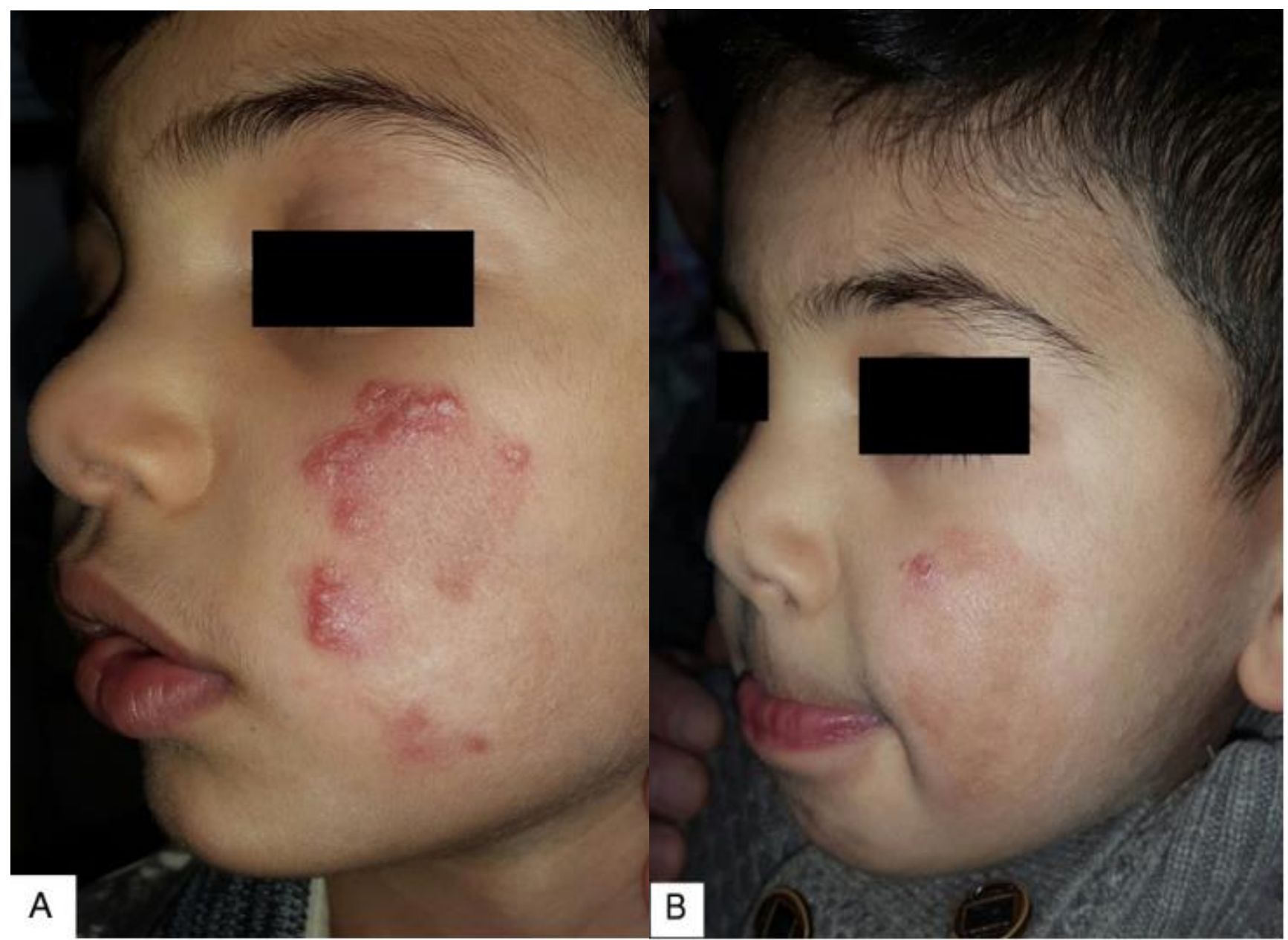

Figure 4. Four years old male with leishmania recidivans showing atrophic patch with active raised arch at the periphery: (A) before treatment and (B) the same patient one month after treatment showing complete clearance of active lesions

\section{DISCUSSION}

Leishmania recidivans (LR), though it is rare, a wellrecognized clinical variant of CL characterized by the development of new papules or nodules in the center or periphery of a healed lesion of leishmaniasis. Reactivation of the persistence parasites in the scarred tissue of $\mathrm{CL}$ is the probable cause for the appearance of LR lesion [24].

The reason for the appearance of LR is not well known. Incomplete treatment course, generation of specific types of immune responses and also strain and species of the causative organism, and defect in the cellular immunity of the host may all contribute to development of LR. Relapses may occur more commonly within two years after the first manifestations of the disease $[6,25,26]$.

The present work had shown that all cases were initially treated with intralesional SSG several times still no clearance of lesions but instead and after many months to few years, atrophy and scarring at the centers of lesions were developed, with active dusky red papules and nodules around or in the site of primary healed lesion sometimes forming rings or arches. These clinical features of LR that are observed by the current work are consistent with what has been reported $[8,27,28]$.
In the present study, the location of lesions was mostly on the face and upper limbs and this is in agreement with other reports $[8,27,28]$.

The age affected in this study was mostly childhood group and this is similar to what seen in other studies $[8,27,28]$.

In reviewing of literature, we found that some patients who received systemic SSG or meglumine antimoniate for the treatment of CL were developed LR $[8,27,29]$ but LR lesions were not reported after intralesional SSG for CL treatment. So this is the first study that recorded the appearance of LR after intralesional SSG .The reason for the development of LR after intralesional SSG is unknown but we can suggest that imperfect treatment as intralesional infiltration was carried only in the center and leaving the periphery ,and or violence injection of lesions will cause the spread of the parasite to the periphery thus escape from drug action or even parasite drug resistance and this followed by reactivation of these parasites thus causing LR.

Zinc is an intracellular signal molecule that plays an essential role in enhancing the function of monocytes, macrophages, dendrocytes and cell-mediated immunity that are in turn effective in body defense mechanism against 
Leishmania. In addition, zinc has the ability of protein precipitation and also acts directly on the enzymatic system of leishmania parasite [30].

Ketoconazole is an antifungal safe drug [22] that inhibits fungal synthesis of ergosterol, a component of fungal cell membranes. As with all azole antifungal agents, ketoconazole acts mainly by inhibiting the enzyme 14-alphademethylase and thus block ergosterol synthesis [31].

The therapeutic use of combination of oral ketoconazole and zinc sulfate yield a solution for the rapid development of resistance and drug to drug interaction in presently new rising antifungal drugs, they act in a synergistic process by:

1. Ketoconazole can interrupt protozoa membrane transport by preventing the proton pump could be improved by the utilization of zinc sulfate [32].

2. Zinc inhibits the enzymes that are essential for the parasite carbohydrate metabolism [33,34].

3. The inhibition of ergosterol biosynthesis carried by ketoconazole could be improved by the usage of zinc sulfate [31].

The probable mechanism of action of podophyllin as an antileishmanial drug could not be well explained but the well-known actions of podophyllin on cells could be applied on leishmania parasites and these actions include: blocking oxidation enzymes in tricarboxylic acid cycle, arresting cellular mitosis in metaphase, inhibiting axonal transport, protein, DNA, and RNA synthesis [35].

In the present work, all patients showed complete clearance of the lesions and the cure rate was $100 \%$ after two months of therapy. These results were comparable to another study [8] in which a combination of allopurinol and meglumine antimoniate was used and the cure rate was $87.5 \%$. This superiority of cure rate in current study could be due to the high efficacy and the synergistic effects of oral ketoconazole and zinc sulfate and topical podophyllin that are used in this work with their antileishmanial activity.

From these different mechanisms of actions as antileishmanial of oral zinc sulfate, oral ketoconazole and topical podophyllin mentioned above, in addition to our experience with these medications in the treatment of different skin disorders, we are encouraged to use these combinations to get a synergistic effect, high cure rate, short duration of treatment, with minimal or no adverse effects.

\section{CONCLUSIONS}

Leishmania recidivans is an important clinical feature of CL with more resistance to the standard treatment regimens and more duration to recover. This work showed that LR is not uncommon type of leishmaniasis with a new explanation for LR evolution as intralesional therapy of CL might induce LR. The mechanism behind this reaction, cannot be well explained but we can speculate that local injection at the center of lesion only but not at the periphery and or the powerful forcing injection might push parasites to move to the periphery, in addition there might be parasite drug resistance. Oral ketoconazole and zinc sulfate combined with topical podophyllin are effective mode of treatment for LR as for ordinary CL.

Author contributions: All authors have sufficiently contributed to the study, and agreed with the results and conclusions.

Funding: No funding source is reported for this study.

Declaration of interest: No conflict of interest is declared by authors.

Ethical statement: Informed consent was taken for each patient or his/her parents before starting the therapy, after a full explaining about the nature of the disease, the method of treatment, complications, and follow up.

\section{REFERENCES}

1. Sharquie KE, Najm RA, Farjou IB, Al-Timimi DJ. Oral zinc sulfate in the treatment of acute cutaneous leishmaniasis. Clin Exp Dermatol 2001;26(1):21-6. doi: 10.1046/j.1365-2230.2001.00752.x.

2. Neouimine NI. Leishmaniasis in the eastern Mediterranean region. East Mediterr Health 2001;2(1):94-101. doi: 10.26719/1996.2.1.94.

3. Markle WH, Makhoul K. Cutaneous leishmaniasis: Recognition and treatment. Am Fam Physician 2004;69(6):1455-60.

4. Ashford RW, Desjeux P, de Raadt P. Estimation of population at risk of infection and number of cases of leishmaniasis. Parasitol Today 1992;8(3):104-5. doi: 10.1016/0169-4758(92)90249-2.

5. WHO. Communicable disease working group on emergencies. WHO Office, Baghdad. 2003:39-44. Available at: www.who.int/diseasecontrol_emergencies/ toolkits/Iraq_profile_ok.pdf

6. Marovich MA, Rosalia L, Marc S, et al. Leishmaniasis recidivans recurrence after 43 years: A clinical and immunologic report after successful treatment. Clin Infect Dis 2001;33:1076-1079. doi: 10.1086/322643.

7. Ekiz O, Rifaioglu EN, Sen BB, Culha G, Ozgur T, Dogramaci AC. Leishmaniasis recidiva cutis of the lips mimicking granulomatous cheilitis. Indian J Dermatol 2015;60(2):216. doi: 10.4103/0019-5154.152576.

8. Esfandiarpour I, Dabiri SH. Treatment of cutaneous leishmaniasis recidivans with a combination of allopurinol and meglumine antimoniate: A clinical and histologic study. Int J Dermatol 2007;46(8):848-52. doi: 10.1111/j.1365-4632.2007.03086.x.

9. Sharquie KE, Hassen AS, Hassan SA, Al-Hamami IA. Evaluation of diagnosis of cutaneous leishmaniasis by direct smear, culture and histopathology. Saudi Med J 2002;23(8):925-8.

10. AlSamarai AM, AlObaidi HS. Cutaneous leishmaniasis in Iraq. J Infect Dev Ctries 2009;3(2):123-9. doi: 10.3855/jidc.59. 
11. Al-Heany AR, Sharquie KE, Al-Najar SA, Noaimi AA. Cutaneous leishmaniasis: Comparative techniques for diagnosis. IOSR J Dent Med Sci 2014;13(4):33-7. doi: 10.9790/0853-13453337.

12. Al-Hucheimi SN, Sultan BA, Al-Dhalimi MA. A comparative study of the diagnosis of Old World cutaneous leishmaniasis in Iraq by polymerase chain reaction and microbiologic and histopathologic methods. Int J Dermatol 2009;48(4):404-48. doi: 10.1111/j.1365-4632.2009.03903.x.

13. Al-Bayatii NS. Diagnostic and epidemiological study of infection with cutaneous leishmaniasis [PhD thesis, University of Tikrit]. 2015.

14. Rahi A. Identification of leishmania species causing cutaneous leishmaniasis using real-time PCR in Iraq. Int J Dev Res 2014;4:1459-62.

15. Sharquie KE, Al-Talib K. Intralesional therapy of cutaneous leishmaniasis with sodium stibogluconate antimony. Br J Dermatol 1988;119(1):53-7. doi: 10.1111/j.1365-2133.1988.tb07100.x.

16. Sharquie KE, Hussian AK, Turki KM. Intralesional therapy of cutaneous leishmaniasis with hypertonic sodium chloride solution. Pan-Arab League Dermatol 1994;5:85-91.

17. Sharquie KE. A new intralesional therapy of cutaneous leishmaniasis with hypertonic sodium chloride. J Dermatol 1995;22(10):732-7. doi: 10.1111/j.13468138.1995.tb03911.x.

18. Sharquie, KE, Al-Azzawi KE. Intralesional therapy of cutaneous leishmaniasis with $2 \%$ zinc sulfate solution. J Pan Arab League 1996;7:41-6.

19. Sharquie KE, Najim RA, Farju IB. A comparative controlled trial of intralesionally administered zinc sulphate, hypertonic sodium chloride and pentavalent antimony compound against cutaneous leishmaniasis. Clin Exp Dermatol 1997;22(4):169-73. doi: 10.1111/j.1365-2230.1997.tb01054.x.

20. Sharquie KE, Noaimi AA, Al-Ghazzi A. Treatment of cutaneous leishmaniasis by topical $25 \%$ podophyllin solution (single, blinded, therapeutic, controlled study). J Dermatology and Dermatol Surg 2015;19(2):108-13. doi: 10.1016/j.jdds.2014.10.001.

21. Sharquie KE, Noaimi AA, Al-Salam WS. Treatment of acute cutaneous leishmaniasis by oral zinc sulfate and oral ketocanazole singly and in combination. J Cosmet Dermatol Sci App 2016;6(3):105-15. doi: 10.4236/jcdsa.2016.63014.

22. Sharquie KE, Noaimi AA, Al-Salam WS. The safety of oral ketoconazole in the treatment of skin diseases (single blinded, therapeutic, comparative study). J Cosmet Dermatol Sci App 2018;8(4):264-71. doi: 10.4236/jcdsa.2018.84028.
23. Sharquie KE, Noaimi AA, Al-Zoubaidi MS. Treatment of basal cell carcinoma by topical $25 \%$ podophyllin solution. J Cosmet Dermatol Sci App 2015;5(3):189-97. doi: 10.4236/jcdsa.2015.53023.

24. Oliveira-Neto MP, Mattos M, Souza CS, Fernandes O, Pirmez C. Leishmaniasis recidiva cutis in new world cutaneous leishmaniasis. Int J Dermatol 1998;37(11):846-9. doi: 10.1046/j.13654362.1998.00478.x.

25. Stefanidou MP, Antoniou M, Koutsopoulos AV, et al. A rare case of leishmaniasis recidiva cutis evolving for 31 years caused by leishmania tropica. Int J Dermatol 2008;47(6):588-9. doi: 10.1111/j.13654632.2008.03240.x.

26. Gomes CM, Damasco FDS, Oliveira de Morais O, Ribeiro de Paula CD, Sampaio RN. Recurrent cutaneous leishmaniasis. An Bras Dermatol 2013;88(3):462-4. doi: 10.1590/abd1806-4841.20131885.

27. Gitaria JW, Nzoub SM , Wamunyokolia F, et al. Leishmaniasis recidivans by leishmania tropica in Central Rift Valley Region in Kenya. Int J Infect Dis 2018;74:109-16. doi: 10.1016/j.ijid.2018.07.008.

28. Sharifi I, Fekri AR, Aflatoonian MR, et al. Leishmaniasis recidivans among school children in Bam, South-east Iran, 1994-2006. Int J Dermatol 2010;49(5):557-61. doi: 10.1111/j.1365-4632.2010.04419.x.

29. Dassoni F, Daba F, Naafs B, Morrone A. Leishmaniasis recidivans in Ethiopia: Cutaneous and mucocutaneous features. J Infect Dev Ctries 2017;11(1):106-10. doi: 10.3855/jidc.8516.

30. Prasad, AS. Zinc: Role in immunity, oxidative stress and chronic inflammation. Curr Opin Clin Nutr Metab Care 2009;12(6):646-52. doi: 10.1097/MCO. ob013e3283312956.

31. Eil, C. Ketoconazole binds to the human androgen receptor. Horm Metab Res 1992;24(8):367-70. doi: 10.1055/s-2007-1003337.

32. Piérard-Franchimont C, Goffin V, Decroix J, Piérard, GE. A multicenter randomized trial of ketoco-nazole $2 \%$ and zinc pyrithione $1 \%$ shampoos in severe dandruff and seborrheic dermatitis. Skin Pharmacol Appl Skin Physiol 2002;15(6):434-41. doi: 10.1159/000066452.

33. Bernstein BE, Hoffman RC, Klevit RE. Sequence-specific DNA recognition by cys2, his2 zinc fingers. Ann N Y Acad Sci 1994;726:92-102; discussion 10-104. doi: 10.1111/j.1749-6632.1994.tb52800.x.

34. Rhodes D, Klug A. Zinc fingers. Sci Am 1993;268:56-65. doi: 10.1038/scientificamerican0293-56.

35. Oslen DG, Dart RC. Skin and mucous membrane agents. nIn: Dart RC, ed. Medical toxicology, 3rd edn. Philadelphia: Walters Kluwer Company, 2004:1003-4. 